\title{
Current Evidence for Nutrition Intervention: A Meta-analysis
}

\author{
Zelalem Tafese $^{1,}$, , Yifru Berhan ${ }^{2}$ \\ ${ }^{1}$ School of Nutrition, Food Science and Technology, Hawassa University, Hawassa, Ethiopia \\ ${ }^{2}$ Consultant at St. Paul's Hospital, Millennium Medical College, Addis Ababa, Ethiopia
}

Email address:

wudasiez@gmail.com (Z. Tafese)

*Corresponding author

\section{To cite this article:}

Zelalem Tafese, Yifru Berhan. Current Evidence for Nutrition Intervention: A Meta-analysis. Journal of Food and Nutrition Sciences. Vol. 9, No. 3, 2021, pp. 73-83. doi: 10.11648/j.jfns.20210903.12

Received: April 8, 2021; Accepted: May 31, 2021; Published: June 7, 2021

\begin{abstract}
Although different combinations of nutrition interventions are employed, most are insufficient to reduce stunting significantly. This study aimed to draw current evidence to reduce stunting among under 5 years children from randomized controlled trials. Eligible randomized controlled trials met inclusion criteria were included. The weighted mean effect sizes with 95\% CIs were used as summary measures for changes in height-for-age $\mathrm{z}$ score (HAZ) using random-effect models; heterogeneity was analyzed using predefined characteristics. From the total of 116 articles, 47 randomized controlled trials with a sample size of 35,115 study participants were included for analysis. Except for educational intervention $(0.14 ; 95 \% \mathrm{CI}$ : $0.00,0.27)$, the weighted mean effects of iron, zinc, water, sanitation and hygiene (WASH), multiple micronutrients (MMN) and dietary interventions were all insignificant. A fixed combination of nutrition interventions demonstrating similar benefit in all contexts is not found. Our results are insufficient to make a recommendation on the most appropriate interventions to reduce stunting in all settings. This result highlights the importance of further evidence before nutrition component formulation for large-scale interventions. The short duration of the interventions and lack of information about the infection status of participants in most of the included trials remain two of the possible limitations needing consideration.
\end{abstract}

Keywords: Evidence, Iron, Meta-analysis, Nutrition Education, Sanitation, Stunting, Zinc

\section{Introduction}

Stunting, wasting, and micronutrient deficiencies, remain one of the major challengesamong children younger than 5 years in developing countries [1]. For effective reduction of stunting, it is vital to have a deeper understanding of factors contributingto linear growth restrictions [2]. Growth restrictions may stem from deficiencies in single nutrients, micronutrients, macronutrients, or more commonly, a combination of many nutritional deficiencies [3].

Globally there are ambitious targets to reduce the prevalence of stunting by $40 \%$ between 2010 and 2025 [4]. Some countries have set even higher goals like the Sekota declaration by the government of Ethiopia, to end all forms of child undernutrition by 2030 [5]. Although undernutrition is commonly associated with food insecurity and hunger, it is unclear which nutrition intervention can be most effective in a specific context, particularly in countries where undernutrition is a continuing problem [6].

As noted by previous studies the causes and consequences of stunting and growth faltering in most developing countries are complex and varied [7-9]. Other studies emphasized stunting in infants and young children is related to many factors, including socioeconomic status, dietary intake, infections, maternal nutritional status, micronutrient deficiencies, and various environmental factors [10, 11]. Because of this, it is unlikely to formulate a single intervention to address child chronic undernutrition sufficiently; rather packages of interventions are needed on the multitude of causes [12]. A prior study noted that at least three key sectors need to engageand contribute to nutrition improvements; the agriculture, health, and environment sectors [13]. With this background, it can be understood that there are numerous ways, both simple and more complex, through which nutrition can be integrated into different sector 
interventions, both at the planning and implementation phase [12]. The Lancet series in 2013 highlighted nutritionsensitive programming as a key element in reducing undernutrition [1], but a multi-dimensional approach, which addresses both dietary and non-dietary factors comprehensively, would represent an important consideration to promote linear growth in early childhood [14].

Although, there are well-established, nutrition-specific, and sensitive interventions [15], most interventions are reported insufficient to reduce stunting particularly in food insecure settings $[16,17]$. A review conducted to synthesize the impacts of nutrition program interventions to reduce malnutrition noted that there was no clear pattern of impacts on indicators across interventions [18].

To address stunting effectively, it is important to understand the timing and the ability of individuals to catch up in terms of linear growth. But, there is a knowledge gap on the causes of undernutrition and the impact of interventions, particularly in the agriculture and environment sectors [12]. Given the significant role of strong evidence in formulating nutrition interventions, there is a need for further evidence particularly for nutrition-sensitive approaches [6]. Similarly, the importance of selected nutrition interventions directed towards the mothers, infants, and young children have been discussed by previous studies, the need for further evidence for conclusions is re-emphasized [19-21]. On the other hand, the vital role of indirect interventions such as behavior change communications accompanied by conditional cash transfers was noted by researchers to reduce stunting $[11,22]$.

Furthermore, other studies underlined the importance of assessing nutrition interventions through the agriculture and environment sectors to address the challenges of malnutrition fully from a multi-sector perspective $[9,13]$. Therefore, the present study aimed to draw further evidence on nutrition interventions to reduce stunting from randomized controlled trials.

\section{Methods}

\subsection{Protocol and Registration}

This review was conducted following the PRISMA-P (Preferred Reporting Items for Systematic Reviews and Meta-Analyses-Protocol) checklist [24]. The protocol is registered with PROSPERO under the registration number: CRD42019118431

\subsection{Search Strategies}

The Preferred Reporting Items for Systematic Reviews and Meta-Analyses (PRISMA) was used to conduct this study. Eligible randomized controlled trials evaluating the effect of micronutrients, dietary, water, sanitation, and hygiene and educational interventions among children under five years, published from 2000 to 2019 were included. The primary outcome was a change in linear growth of children as measured by HAZ following a specific type of nutrition intervention; studies in which HAZ was not reported were excluded.

An electronic search of Medline, Pub Med, Health Internetwork Access to Research Initiative (HINARI), Google Scholar databases, and the Cochrane Library published up to 2019 were conducted. The search was done using specific keywords: ("stunting" OR "growth faltering among children" OR chronic undernutrition reduction OR approach among children AND "determinant factors" OR evidence-based nutrition intervention "micronutrient", "nutrition education" "water, sanitation and hygiene" "Iron" "zinc" "dietary intervention") AND (Randomized control trials). A function extracting related articles was used during the search process. The full versions of the English-language analyzed articles of found papers were used during the selection process (Figure 1).

Selection process

The research question was framed using the PICO-ST (participant, intervention, comparator, outcome, study design, and time frame). The criteria of article selection were study participants children (under five years), both sexes, all ethnicity $(\mathrm{P})$; Intervention to reduce stunting (I); Educational, dietary, micronutrient, and WASH interventions (C); Reporting the linear growth of the child as measured by mean $\pm \mathrm{SD}$ (HAZ) in the results of the study (O); Randomized controlled trials (S); and Published from 2000 up to 2019 (T).

After searching the keywords in the mentioned databases, selected studies were imported to the Mendeley reference management software, and duplicates were deleted. The title and abstracts of the remaining articles were studied by two researchers separately and those studies that did not meet the mentioned criteria were excluded. Then, the full text of the potentially relevant papers was retrieved and read for data extraction, quality assessment, and meta-analysis. In the cases of disagreements between the two researchers on an article, discussions were made to reach an agreement. The articles excluded during each screening phase as well as reasons for study exclusion during the second phase were described using a flow diagram following the PRISMA statement [24].

\subsection{Methodological Quality Assessment}

Studies were assessed for methodological quality using the quality assessment tool for quantitative studies developed by the Effective Public Health Practice Project [25]. The tool has six components (selection bias, study design, confounders, blinding, data collection method, withdrawals, and dropouts). With this validated instrument a global rating as strong (no weak rating), moderate (one weak rating), or weak (two or more weak ratings) was assigned to each study based on the criteria described in the EPHPP dictionary.

\subsection{Data Extraction}

Key descriptive data, including the sample size for intervention and control, location of intervention, characteristics of the study participants, dose and duration of 
the intervention (when applicable), and HAZ as an outcome of interest were extracted into a standardized form. The reported sample size corresponds to the number of subjects who completed the intervention. The included studies were grouped into six intervention categories: 1) Iron, 2) Zinc, 3) MMN ( $\geq 3$ micronutrients), 4) dietary, 5) WASH and 6) Educational interventions. Studies with $>1$ intervention group were treated as independent studies.

\subsection{Summary Measures}

The mean difference of change in HAZ between the experimental and control groups or before and after a specific intervention with a $95 \%$ confidence interval was considered as a summary measure.

\subsection{Data Synthesis}

The data from the included studies were analyzed by a metaanalysis statistical approach using RevMan analytic software (version 5.0.25), an inverse variance method. $\mathrm{I}^{2}$ statistics were used to measure the heterogeneity of the studies, using a random model meta-analysis [25]. The funnel plots were visually assessed to investigate potential publication bias [26]. Sensitivity analysis as done as noted by a previous study [27], to assess the effect of one or more influential studies on the outcome, by excluding studies using characteristics of the comparator: (placebo or other treatment as a comparator group), characteristics of the intervention: (range of doses used for treatment), characteristics of participants (age $<5$ yrs or not) and location of the study (LMICs or not).

\section{PRISMA 2009 flow diagram}

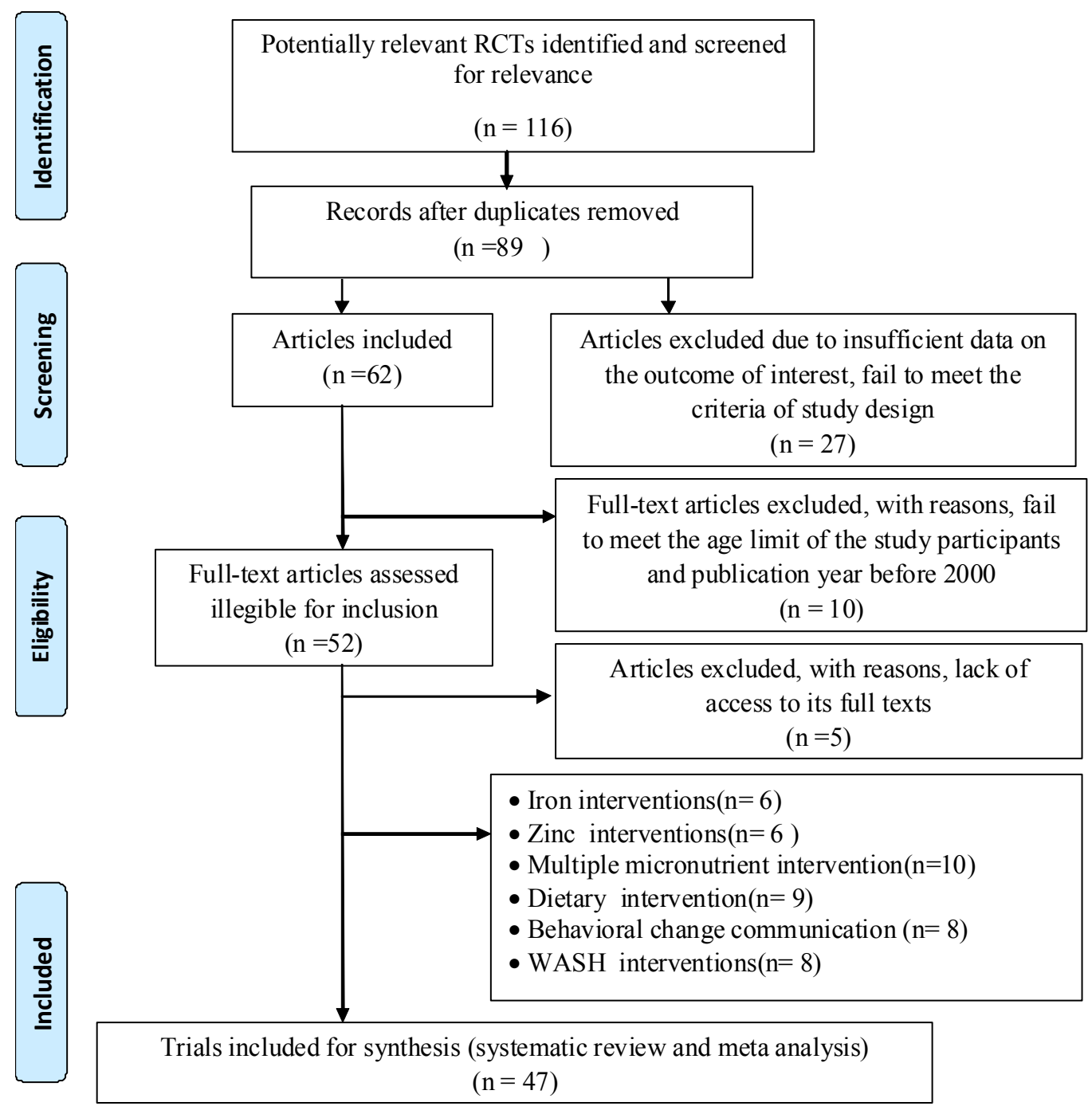

Figure 1. The PRISMA chart used to select the studies for the present review Adopted from Moher et al. 2009 [23].

\section{Results}

During the initial search, 116 articles were retrieved.
Next, 27 articles were removed as they were duplicates (based on author name, article title, year of publication, and journal name) and unrelated articles; 27 more articles were excluded due to insufficient data on the outcome of interest. 
The full texts of 62 articles were read and 15 articles were deleted due to the age limit of the study participants and lack of access to its full texts (Figure 1). In this way, 47 studies all RCTs with a total sample size of 35,115 participants (supplemental docs), investigating the effects of the six major packages of interventions on the outcome of interest were evaluated. The numbers of study participants range from 28 [28] to 3046 [29]. The selected trials reported the effects of specific nutrition interventions on HAZ in low and middle-income countries (LMICs) mostly in food-insecure settings. Most included studies were of strong quality. Moderate quality was assessed for four studies, and one study assessed as weak (Supplemental Table 2).

\subsection{Iron Supplementation}

A total of six studies were included in the meta-analysis. The number of participants in each study ranged from 28 to 75 and all were under five years of age. The dose of iron ranged from 1 to $60 \mathrm{mg} / \mathrm{d}$. Except in one study [30], the daily dose of iron was related to deficiency level and the bodyweight of the child. The duration of supplementation lasted from 3 to 6 mo. Effect sizes for change in HAZ ranged from -0.94 to 0.23 . There was significant heterogeneity $(\mathrm{P}=0.001)$. The overall mean effect was not statistically significant -0.12 (95\% CI: $0.39,0.14$ ) (Figure 2). Sensitivity analyses excluding the study with the highest doses of iron used for treatment [28] -0.15 (95\% CI: $-0.52,0.23)$ did not alter the conclusions.

\subsection{Zinc Supplementation}

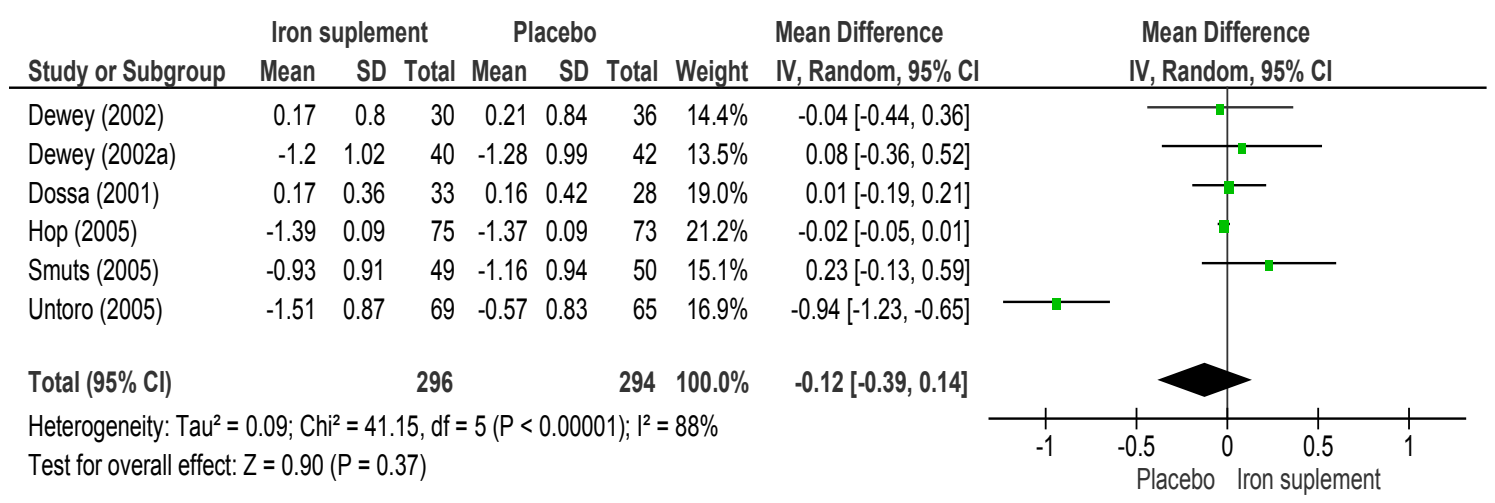

Figure 2. Forest plots for the effect iron supplement on HAZ of children under five years.

Six studies were included for analysis with the number of participants ranging from 64 to 1193, all less than five years. The dose of zinc ranged from 7 to $15 \mathrm{mg} / \mathrm{d}$ with a 4 to $10 \mathrm{mo}$ supplementation duration. Effect sizes for change in HAZ ranged from -0.02 to 0.46 . Most of the studies (83\%) reported a positive effect size, even though the overall mean effect was statistically insignificant $(0.03 ; 95 \%$ CI:-0.03, 0.08 ) (Figure 3). Sensitivity analyses excluding studies in which zinc supplementation was more than $10 \mathrm{mg}(0.05$; 95\% CI:-0.02, 0.12) did not alter the conclusions.

Mean Difference Mean Difference

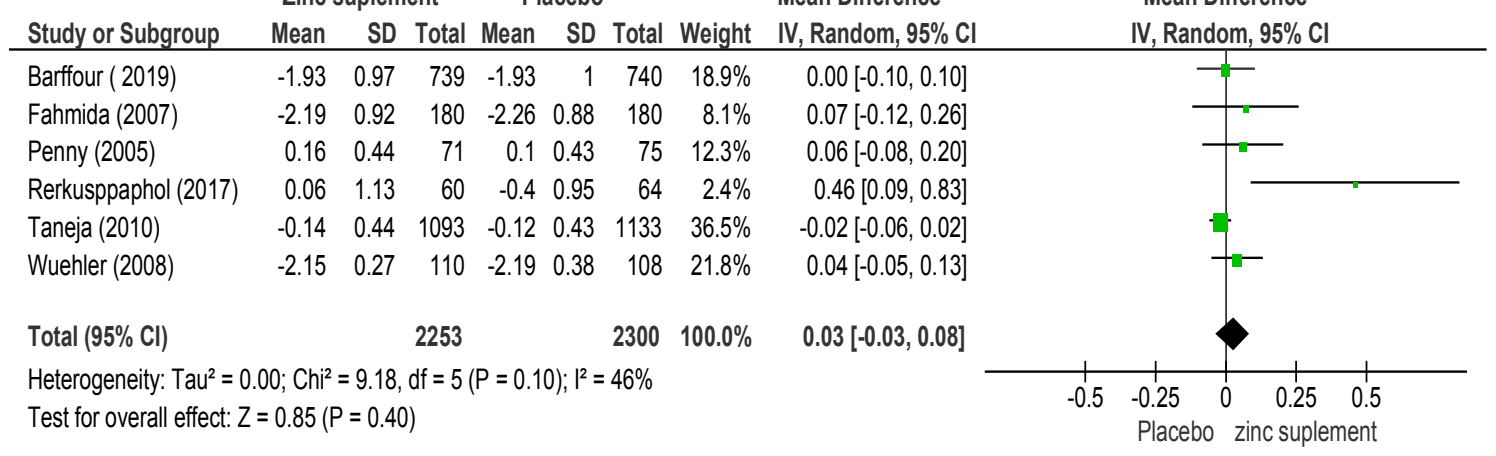
IV, Random, $95 \% \mathrm{Cl}$

Figure 3. Forest plots for the effect zinc supplement on HAZ of children under five years.

\subsection{MMN Supplementations}

A total of 10 studies were included in the analysis. The number of study participants ranged from 49 to 267. All interventions contained zinc and iron with a study duration lasting from $23 \mathrm{wk}$ to 6 mo. Effect sizes for change in HAZ ranged from -0.94 to 0.35 , and there was significant heterogeneity ( $\mathrm{P}=0.001)$. The overall mean effect was not statistically significant (-0.07; 95\% CI:-0.20, 0.06) (Figure 4). Sensitivity analyses excluding a study that used another treatment as a comparator group [31] $(-1.0 ; 95 \% \mathrm{CI}:-0.24$, 0.03 ) did not alter the conclusions. 


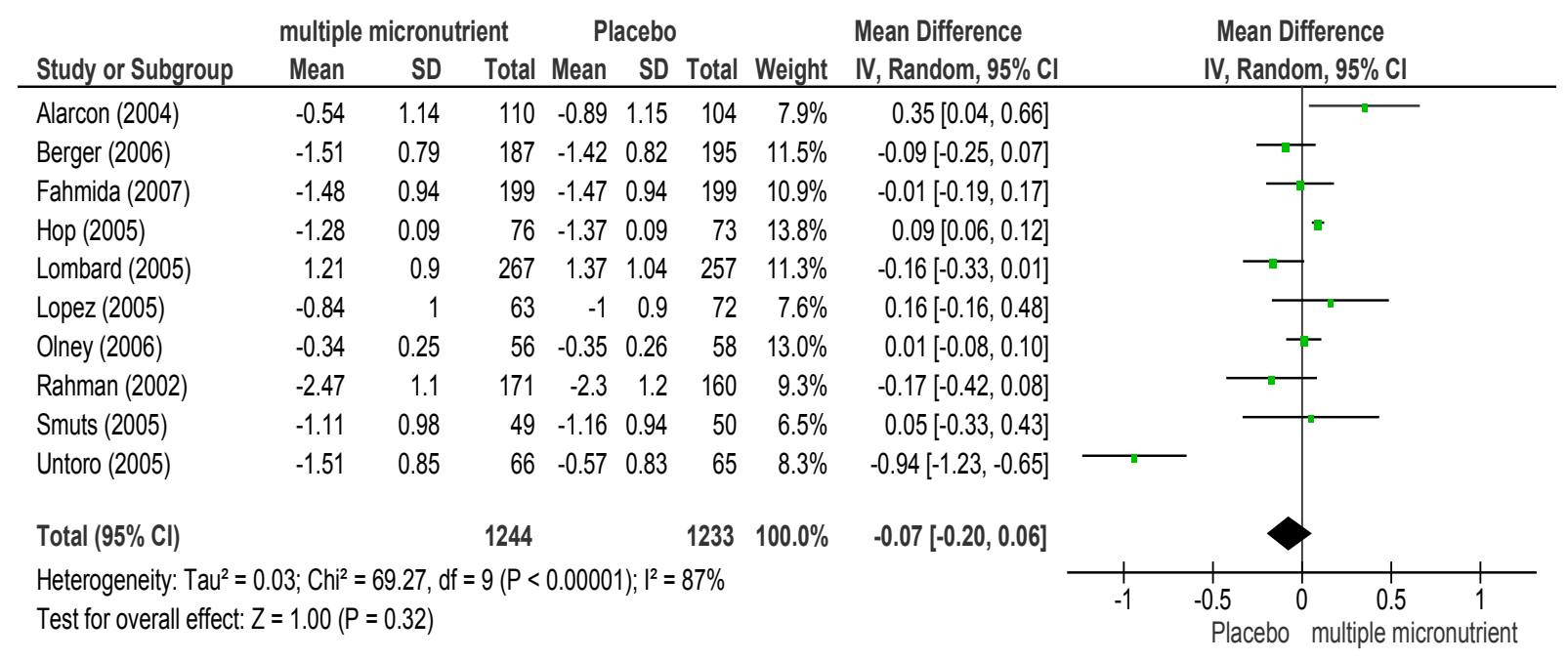

Figure 4. Forest plots for the effect MMN supplement on HAZ of children under five years.

\subsection{Dietary Intervention}

Nine studies that examined the impact of complementary food interventions on the linear growth of children were identified and included in the meta-analysis. All data were obtained from studies conducted in LMICs. The number of subjects ranged from 30 to 1312 and study participants in all studies were less than $5 \mathrm{yr}$ except in one study which was 3- 6yrs [32]. The complimentary food was supplemented daily in all studies and the duration of intervention lasted from 6 to 18 mo. Effect sizes for change in HAZ ranged from -0.38 to 0.60 . There was significant heterogeneity $(P=0.0002)$. The overall mean effect was not statistically significant $(0.01$; 95\%CI: -0.08, 0.06) (Figure 5). Sensitivity analyses excluding the study with an age range of more than 5 years ($0.05 ; 95 \%$ CI: $-0.12,0.03$ ) but did not alter the conclusions.

\begin{tabular}{|c|c|c|c|c|c|c|c|c|c|}
\hline \multirow[b]{2}{*}{ Study or Subgroup } & \multicolumn{3}{|c|}{ New complementary food } & \multicolumn{3}{|c|}{ usual diet } & \multirow[b]{2}{*}{ Weight } & \multirow{2}{*}{$\begin{array}{l}\text { Mean Difference } \\
\text { IV, Random, } 95 \% \mathrm{Cl}\end{array}$} & \multirow{2}{*}{$\begin{array}{c}\text { Mean Difference } \\
\text { IV, Random, } 95 \% \mathrm{Cl}\end{array}$} \\
\hline & Mean & SD & Total & Mean & SD & Total & & & \\
\hline Adu-afarwuah (2007) & -0.14 & 1.22 & 97 & -0.74 & 0.95 & 81 & $11.1 \%$ & $0.60[0.28,0.92]$ & \\
\hline Christian (2015) & -1.43 & 1.1 & 789 & -1.33 & 1.04 & 1312 & $16.1 \%$ & $-0.10[-0.20,-0.00]$ & \\
\hline lannotti (2017) & -1.39 & 1.35 & 75 & -1.71 & 1 & 73 & $9.6 \%$ & $0.32[-0.06,0.70]$ & \\
\hline Ihab (2014) & -1.46 & 0.62 & 30 & -1.58 & 0.72 & 30 & $10.6 \%$ & $0.12[-0.22,0.46]$ & \\
\hline Ihab (2014a) & -1.96 & 1.12 & 30 & -1.58 & 0.72 & 30 & $7.8 \%$ & $-0.38[-0.86,0.10]$ & \\
\hline John (2008) & -0.59 & 1.22 & 54 & -0.74 & 0.95 & 57 & $9.1 \%$ & $0.15[-0.26,0.56]$ & \\
\hline Krebs (2012) & -2.04 & 1.33 & 532 & -1.89 & 1.42 & 530 & $14.8 \%$ & $-0.15[-0.32,0.02]$ & \\
\hline Lopriore (2004) & -2.68 & 0.59 & 106 & -3 & 0.68 & 45 & $13.3 \%$ & $0.32[0.09,0.55]$ & \\
\hline Olaya (2013) & -1.12 & 1.1 & 38 & -1.02 & 1 & 38 & $7.8 \%$ & $-0.10[-0.57,0.37]$ & \\
\hline Total $(95 \% \mathrm{Cl})$ & & & 1751 & & & 2196 & $100.0 \%$ & $0.09[-0.09,0.27]$ & \\
\hline $\begin{array}{l}\text { Heterogeneity: } \operatorname{Tau}^{2}= \\
\text { Test for overall effect: } 2\end{array}$ & $\begin{array}{l}5 ; \mathrm{Chi}^{2}=3 \\
0.98(\mathrm{P}=\end{array}$ & $3, \mathrm{df}=$ & $<0.00$ & & & & & & $\begin{array}{cccc}-0.5 & -0.25 & 0 & 0.25 \\
& \text { Usual diet } & \text { New comp }\end{array}$ \\
\hline
\end{tabular}

Figure 5. Forest plots for the effect dietary intervention on HAZ of children under five years.

\subsection{Nutrition Education Interventions}

Eight trials were included for the Meta-analysis and all studies were conducted primarily in LMICs. Participants in each study ranged from 97 to 488 and the age of subjects ranging from birth to 59 mo. The intervention was administered to the child's mother. The duration of intervention ranged from 5 to $12 \mathrm{mo}$. The effect sizes for change in HAZ ranged from -0.35 to 0.41 (Figure 6). Most datasets $(88 \%)$ had a positive effect size, and three were statistically significant. There was significant heterogeneity $(\mathrm{P}<0.00001)$. One study [33] was identified as a possible outlier because of extreme effect sizes. The overall weighted mean effect was found positive; 0.14 (95\% CI: $0.00,0.27)$ and 0.17 (95\% CI: $0.04,0.30)$ with and without the potential outlier. 


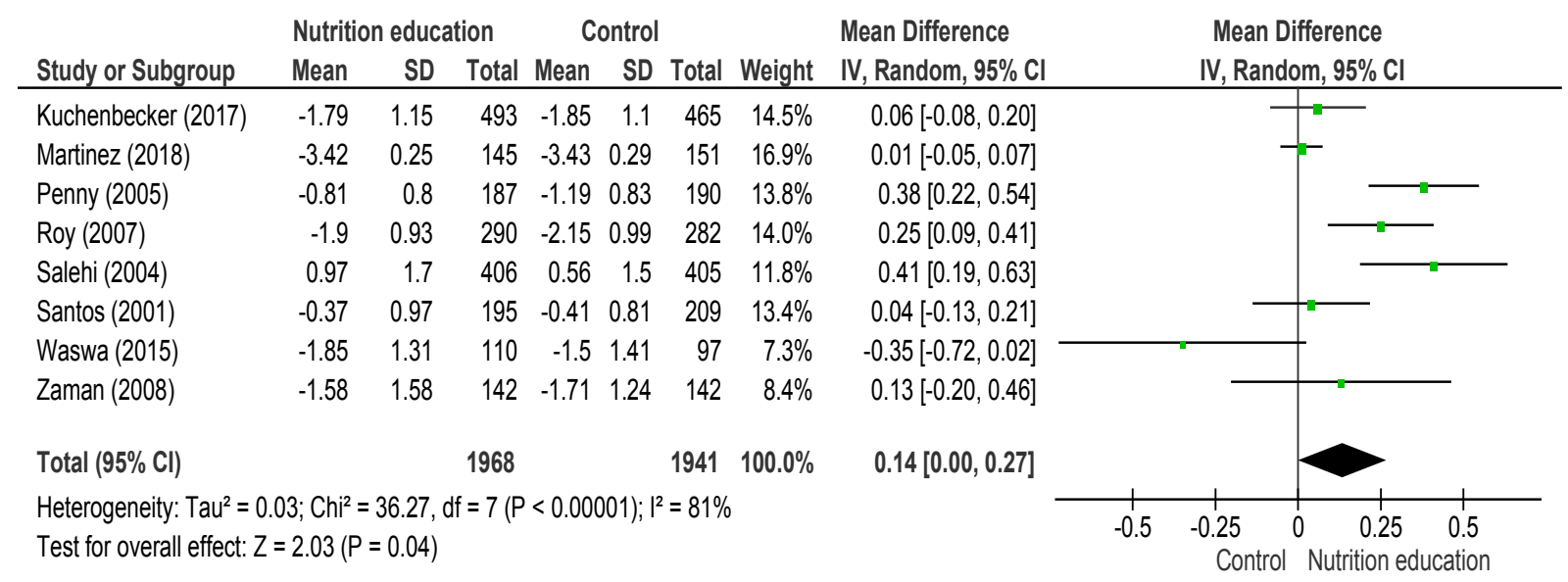

Figure 6. Forest plots for the effect educational intervention on HAZ of children under five years.

\subsection{Water Sanitation and Hygiene Intervention}

Eight randomized trials were identified all conducted in LMICs. The number of subjects in each study ranged from 570 to 3046 and age ranged from birth to $59 \mathrm{mo}$. WASH interventions were administered as water treatment, proper toilet utilization, and hand washing. The duration of intervention ranged from 18 mo to 5 years. The effect sizes for change in HAZ ranged from -0.07 to 0.22 (Figure 7). The majority of datasets $(63 \%)$ had a positive effect size, and two were statistically significant. There was significant heterogeneity $(\mathrm{P}<0.00001)$ and the overall weighted mean effect was 0.05 ( $95 \% \mathrm{CI}:-0.03,0.13$ )

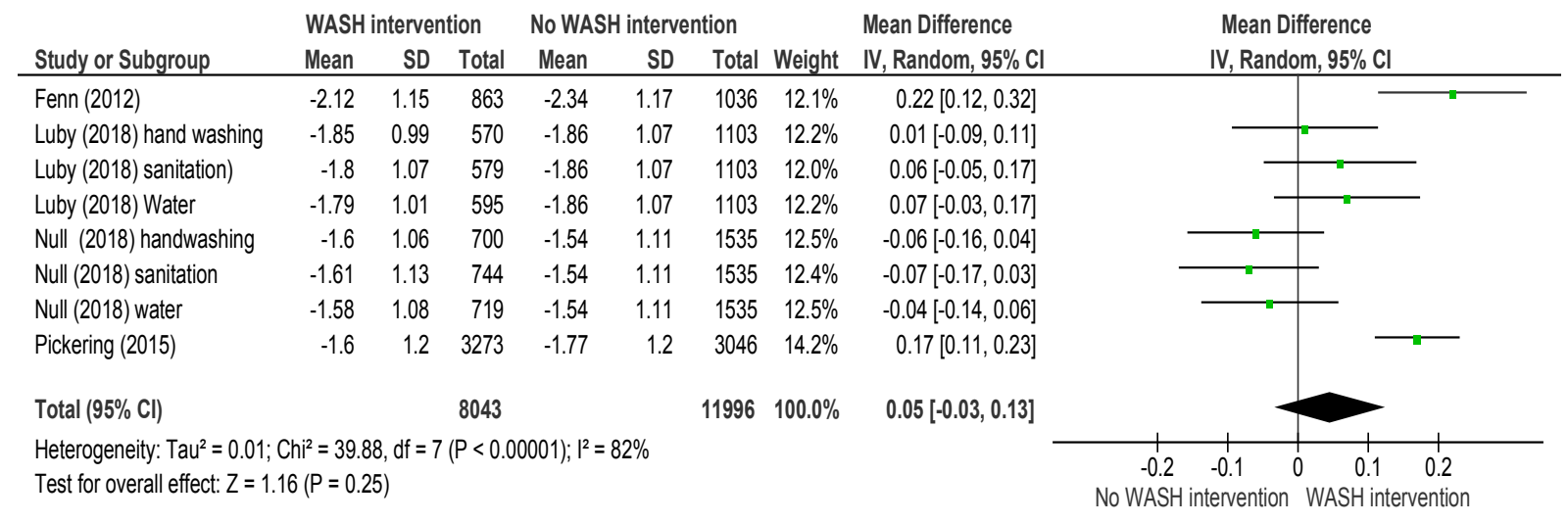

Figure 7. Forest plots for the effect WASH intervention on HAZ of children under five years.

\section{Discussion}

The key findings of this review are summarized in Table 2 (Supplemental docs). The individual interventions of iron, zinc, MMN containing iron and zinc, complimentary food, and WASH did not result in improved linear growth of children under five years of age, whereas interventions providing nutrition education had a significant positive effect $(0.14 ; 95 \%$ CI: $0.00,0.27)$ on linear growth. A range of sensitivity analyses using different assumptions for the change in HAZ did not alter these conclusions. Previous studies have reported that associations between irondeficiency anemia and impaired linear growth may stem from impaired immunity, decreased appetite, and thyroid hormone metabolism [27, 34-35]. Several previous reviews and metaanalyses [36-39] reported an impact of iron supplementation on the linear growth of children. Even though iron supplementation is one of the most widely practiced nutrition interventions, except for one study [28]; most of the trials in our review did not show a positive impact of iron supplementation on the linear growth of children [32, 40-42]. This indicates that iron supplementation as a single intervention is insufficient to improve the linear growth of children. Our analysis also showed inconsistent results in different settings. In line with previous studies the effects of iron supplementation on child health varied by the extent of malaria and iron deficiency [43]. Despite methodological differences, our findings were consistent with previous reviews $[27,36,44]$ and contribute to the growing body of evidence suggesting that iron supplementation as a single intervention is ineffective in promoting the linear growth of children. 
Table 1. Summary estimates for the weighted mean effect sizes of HAZ (95\% CIs) for each intervention (supplemental docs).

\begin{tabular}{|c|c|c|c|c|c|c|}
\hline Outcome & Iron & Zinc & $\mathbf{M M N}^{\mathrm{a}}$ & DI $^{\mathbf{b}}$ & WASH & $\mathbf{N E}^{\mathrm{c}}$ \\
\hline $\mathrm{n}$ & 590 & 4553 & 2477 & 3947 & 20039 & 3909 \\
\hline HAZ & $-0.12(-0.39,0.14)$ & $0.03(-0.03,0.08)$ & $-0.07(-0.20,0.06)$ & $0.09(-0.09,0.27)$ & $0.05(-0.03,0.13)$ & $0.14(0.00,0.27)$ \\
\hline
\end{tabular}

$\mathrm{MMN}^{\mathrm{a}}$-Multiple micronutrientCF${ }^{\mathrm{b}}$ - Dietary interventionNE ${ }^{\mathrm{c}}-\mathrm{Nutrition}^{-}$education

Table 2. Quality rating of the included randomized control trials according to effective public health practice projects qualitative assessment tool for quantitative studies.

\begin{tabular}{|c|c|c|c|c|c|c|c|}
\hline Author/year & $\begin{array}{l}\text { Selection } \\
\text { bias }\end{array}$ & Study design & $\begin{array}{l}\text { Confound } \\
\text { ers }\end{array}$ & Blinding & $\begin{array}{l}\text { Data } \\
\text { collection }\end{array}$ & $\begin{array}{l}\text { Withdrawals } \\
\text { and drop out }\end{array}$ & $\begin{array}{l}\text { Overall quality } \\
\text { assessment }\end{array}$ \\
\hline Adu-afarwuah et al. (2007) & 2 & 1 & 3 & 2 & 1 & 1 & Moderate \\
\hline John et al. (2008) & 1 & 1 & 1 & 1 & 1 & 1 & Strong \\
\hline Alarcon et al. (2004) & 1 & 1 & 1 & 1 & 1 & 1 & Strong \\
\hline Rahman et al. (2002) & 1 & 1 & 2 & 2 & 1 & 1 & Strong \\
\hline Berger et al. (2006) & 1 & 1 & 1 & 1 & 1 & 1 & Strong \\
\hline Olney K et al. (2006) & 1 & 1 & 1 & 1 & 1 & 1 & Strong \\
\hline Lópezet al. (2005) & 1 & 1 & 2 & 2 & 1 & 1 & Strong \\
\hline Rerksuppaphol et al.(2017) & 1 & 1 & 1 & 1 & 1 & 1 & Strong \\
\hline Penny et al. (2004) & 1 & 1 & 1 & 1 & 1 & 1 & Strong \\
\hline Taneja et al. (2010) & 1 & 1 & 1 & 1 & 1 & 1 & Strong \\
\hline Fahmida et al. (2007) & 1 & 1 & 2 & 2 & 1 & 1 & Strong \\
\hline Wuehler et al. (2008) & 1 & 1 & 1 & 1 & 1 & 1 & Strong \\
\hline Barffour et al. (2019) & 1 & 1 & 1 & 1 & 1 & 1 & Strong \\
\hline Smuts et al. (2005) & 1 & 1 & 1 & 1 & 1 & 1 & Strong \\
\hline Dewey et al. (2002) & 2 & 1 & 3 & 2 & 1 & 1 & Moderate \\
\hline Hallamaa et al. (2018) & 1 & 1 & 1 & 1 & 1 & 1 & Strong \\
\hline Fennet al. (2012) & 1 & 1 & 3 & 1 & 1 & 1 & moderate \\
\hline Keenan et al. (2019) & 1 & 1 & 2 & 2 & 1 & 1 & Strong \\
\hline Christian et al. (2015) & 1 & 1 & 1 & 1 & 1 & 1 & Strong \\
\hline Iannotti et al. (2017) & 1 & 1 & 1 & 1 & 1 & 1 & Strong \\
\hline Olaya et al. (2013) & 2 & 1 & 1 & 1 & 1 & 1 & Strong \\
\hline Hadi et al. (2000) & 1 & 1 & 1 & 1 & 1 & 2 & Strong \\
\hline Dossa et al. (2001) & 1 & 1 & 2 & 1 & 1 & 1 & Strong \\
\hline Khan et al. (2011) & 1 & 1 & 1 & 2 & 2 & 1 & Strong \\
\hline Krebs et al. (2012) & 2 & 3 & 1 & 1 & 1 & 1 & Moderate \\
\hline Masudaet al. (2019) & 1 & 1 & 1 & 1 & 2 & 1 & Strong \\
\hline Lopriore et al. (2004) & 1 & 1 & 1 & 1 & 1 & 1 & Strong \\
\hline Ihabet al. (2014) & 1 & 1 & 1 & 1 & 1 & 1 & Strong \\
\hline Nikièma et al. (2017) & 1 & 1 & 1 & 1 & 1 & 1 & Strong \\
\hline Nair et al. (2017) & 1 & 1 & 1 & 2 & 1 & 1 & Strong \\
\hline Penny et al. (2005) & 1 & 2 & 1 & 2 & 1 & 1 & Strong \\
\hline Zaman et al. (2008) & 1 & 1 & 3 & 1 & 3 & 1 & Weak \\
\hline Royet al.(2007) & 1 & 1 & 1 & 1 & 1 & 1 & Strong \\
\hline Waswa et al. (2015) & 1 & 1 & 2 & 2 & 1 & 1 & Strong \\
\hline Salehi et al. (2004) & 1 & 1 & 1 & 1 & 1 & 1 & Strong \\
\hline Santos et al. (2001) & 1 & 1 & 1 & 1 & 1 & 1 & Strong \\
\hline Pickeringet al. (2015) & 1 & 1 & 1 & 1 & 1 & 1 & Strong \\
\hline Luby et al. (2018) & 1 & 1 & 1 & 1 & 1 & 1 & Strong \\
\hline Null et al. (2018) & 1 & 1 & 1 & 1 & 1 & 1 & Strong \\
\hline Lombard et al. (2005) & 1 & 1 & 1 & 1 & 1 & 1 & Strong \\
\hline
\end{tabular}

Similarly, studies have noted associations between zinc deficiency and impaired linear growth associated with impaired immunity and appetite of the child, resulting in short stature in childhood and adult life too, it is also an essential micronutrient that has a vital role in the linear growth of children particularly on bone formation $[45,46]$. With this regard, it is expected that moderate-to-severe zinc deficiency in children resulted in growth faltering. For this reason, the effect of zinc supplementation on linear growth has been a focus of previous meta-analyses [7, 36, 47, 48, 45]. In our review even though the final effect of zinc on linear growth was not statistically significant, most of the individual trials showed the positive effect of zinc on the linear growth of children.

In contrast, other systematic reviews [47, 45] reported a significant effect of zinc supplementation on the linear 
growth of children; of course, there is considerable variability in inclusion criteria compared with our review. The result of our analysis suggests that zinc supplementation does not have a consistent effect on the growth of children when implemented in different settings. Possibly the difference in the effect of the intervention might arise from the variation in other related intervention components in different intervention areas; which results in the change in the overall effect of a particular intervention.

The overall mean effect of MMN interventions was not significant on the linear growth of children $<5 \mathrm{y}$ of age in a random effect model. Previous studies reported inconsistent effects of MMN on the linear growth of children. Some reported non-significant effects of MMN compared to single micronutrients [7, 28, 49-51]; and other studies contradicted these findings [36, 52].

We have limited information on how specific study settings and individual factors influence the impact of MMN interventions. As noted by one study the magnitude of the effect of MMN interventions may vary by the background prevalence of different micro-nutrient deficiencies or infections [36]. Another previous randomized trial reported better effects on linear growth when MMN was supplemented with fat-based food than with usual homeprepared complementary foods [49]. This shows the effect modification of fat-based complimentary food on micronutrient supplementation above and beyond the usual complimentary food in a specific context. Taking all into account, together with other nutrition interventions MMN supplementations should be promoted as a holistic strategy to address stunting particularly in food insecure settings where the dietary quality is low. Overall the findings of our analysis reemphasize the importance of evidence in a specific context to clarify the potential effects of nutrition intervention packages before formulating nutrition program implementations.

Growth faltering has been associated with overall poor diet quality, mainly low intake of animal source foods [53]. However, the results from our review suggest that the overall mean effect of modifying complimentary food was not statistically significant to improve the linear growth of children. Our analysis was limited by the small number of studies ( $n=9)$ and by significant heterogeneity, which is not surprising because food-based interventions can take many forms. Some of the trials use fortified complementary food, some of them animal source foods, and others used new complementary feeding guidelines. Except for two trials [32, 49], most individual trials in our review showed the insignificant result of complementary feeding intervention on the linear growth of children. These might be due to the shorter duration of interventions and length of follow-up of the included studies. Stunting results from long-term nutritional insult; the short duration of most trials $(6 \mathrm{mo})$ may not be long enough to observe a significant effect on the height of the child. The positive effects in most cases may result from the growth-promoting effects of fat-based fortified complementary food.
On the other hand, proper child feeding practices to prevent malnutrition among children could be enhanced by providing nutrition education for caregivers to appropriately feed their children with safe and adequate complementary foods [33]. The present review showed the overall mean effect of nutrition education to be statistically significant; even though the results had significant heterogeneity. Most likely it is because nutrition education interventions are different in approach, frequency, and duration. In our review considerable variations were observed in the types of educational messages delivered: demonstration of recipe preparation, community-based mass intervention or individual counseling, testing the effect of training the nutrition educators, or evaluating effects of nutrition education models. Our analysis suggests that educational interventions can improve feeding practices which lead to improved growth outcomes if complemented with other nutrition interventions.

An association between WASH interventions and the linear growth of children has been reported [54-56]. Most of the individual trials in our review showed a positive impact of WASH intervention on the linear growth of children [21, 29]. But the overall mean effect was of WASH was not statistically significant for improved linear growth. This shows that WASH as a single intervention is not sufficient to improve linear growth, but, our review suggests WASH interventions as one of the main strategies to reduce stunting in poor settings if complemented with other evidence-based interventions. Despite the good quality of most included trials; the shorter duration of intervention and lack of information about the infection status of study participants by most of the trials remain among possible limitations that need consideration.

\section{Conclusion}

In conclusion, nutrition education intervention provided significant positive effects on the linear growth of children. However, the results of the present analysis are insufficient to make a recommendation concerning the most appropriate intervention components to improve the linear growth of children in all settings. Even though a combination of interventions results in greater improvements in the linear growth of children in some settings, which combination of nutrition interventions most effectively reduce stunting in a specific context is unclear. Therefore to achieve significant effects on the reduction of stunting, particularly in food insecure settings where the magnitude of chronic undernutrition is high, evidence about the specific context is vital before nutrition program formulation. Furthermore, this approach enables efficient resource utilization. Factors contributing to stunting are present even before a child is born; therefore, strategies must be focused on a life course approach and these strategies should get due attention by nutrition program implementers and policymakers.

The findings of our study are supporting evidence for the WHO recommendation of considering contextual factors on 
nutrition program implementation for significant reductions in stunting prevalence. Our results also highlighted a need for further trials with longer durations and extended periods of subject follow-up.

\section{Funding}

This research received no specific grant from any funding agency in the public, commercial, or not-for-profit sectors.

\section{Availability of Data and Materials}

The datasets analyzed during the current study are available from the corresponding author on reasonable request.

\section{Authors' Contributions}

ZT devised the main conceptual ideas, article selection, performed the analysis, and wrote the paper, SH involved in article selection, analysis, and review of the manuscript, YB and $\mathrm{AA}$ involved in analysis and reviewing the manuscript.

\section{Conflicting Interests}

None Declared.

\section{Consent for Publication}

The manuscript is submitted exclusively for publication in SAGE nutrition and health journal. We confirm the contents of the manuscript have not been copyrighted or published previously and not now under consideration for publication elsewhere.

\section{Ethical Approval}

The study has been approved by the Institutional Review Board (IRB) of Hawassa University, College of Medicine and Health Sciences.

\section{Acknowledgements}

We would like to thank Hawassa University for technically supporting this research project. We also would like to extend our gratitude to Professor Barbara J Stoecker (Oklahoma State University) for her unreserved help.

\section{References}

[1] Bhutta ZA, Das JK, RizviA, et al. Evidence-based interventions for improvement of maternal and child nutrition: what can be done and at what cost? The Lancet, 2013382 (9890): 452-477.

[2] Prado EL, Yakes Jimenez E, Vosti S, et al. (2019) Path analyses of risk factors for linear growth faltering in four prospective cohorts of young children in Ghana, Malawi, and
Burkina Faso. BMJ Global Health, 2019. 4 (1): 1-11.

[3] Lundeen EA, Behrman JR, Crookston BT, et al. Growth faltering and recovery in children aged 1-8 years in four lowand middle-income countries: Young Lives. Public Health Nutrition 2014, 17 (9): 2131-2137.

[4] World Health Organization. Reducing stunting in children: equity considerations for achieving the Global Nutrition Targets 2025.2018. WHO. Geneva.

[5] Federal Democratic Republic of Ethiopia. The 'Seqota' declaration implementation plan (2016-2030); summary of programme approach document. 2016. Federal Democratic Republic of Ethiopia. Adiss Ababa.

[6] Bush A and Keylock J. Strengthening integration of nutrition within health sector programmes an evidence-based planning resource. European commission directorate general international cooperation and development. 2018. Brussels.

[7] Bhandari N, Bahl R and TanejaS. Effect of micronutrient supplementation on linear growth of children. British Journal of Nutrition, 2001; 85 (S2): S131.

[8] Prendergast AJ and Humphrey JH. The stunting syndrome in developing countries. Paediatrics and International Child Health, 2014; 34 (4): 250-265.

[9] Bhutta ZA, Ahmed T, Black RE, et al. What works? Interventions for maternal and child undernutritionand survival. The Lancet, 2008; 371 (9610): 417-440.

[10] deOnis $\mathrm{M}$ and Branca F. Childhood stunting: a global perspective. Maternal \& Child Nutrition, 2016; 12: 12-26.

[11] Horton S, Shekar M. and Ajay M. Scaling Up Nutrition. 2009. The World Bank. doi: 10.1596/978-0-8213-8077-2.

[12] Reinhardt K and Fanzo J. Addressing chronic malnutrition through multi-sectoral sustainable approaches: a review of the causes and consequences. Frontiers in nutrition, 2014; 1 (13): $1-11$.

[13] Mabhaudhi T, Chibarabada Tand Modi A. Water-foodnutrition-health nexus: Linking water to improving food, nutrition and health in sub-saharan Africa. Int. J. Environ. Res. And Public Health, 2016; 13 (107): 1-19.

[14] Mohammed SH, Habtewold TD, Tegegne BS, et al. Dietary and non-dietary determinants of linear growth status of infants and young children in Ethiopia: Hierarchical regression analysis. PLOS ONE, 2019; 14 (1): e0209220.

[15] de Onis M, Dewey KG, Borghi E, et al. The World Health Organization's global target for reducing childhood stunting by 2025: rationale and proposed actions. Maternal \& Child Nutrition, 2013; 9 (2): 6-26.

[16] FAO, UNICEF, IFAD, WFP, WHO. The State of Food Security and Nutrition in the World. Safeguarding against economic slowdowns and downturns. 2019. Rome.

[17] Hossain M, Choudhury N, Adib Binte Abdullah K, et al. Evidence-based approaches to childhood stunting in low and middle income countries: a systematic review. Archives of Disease in Childhood, 2017; 102 (10): 903-909.

[18] Gillespie S, Menon P, Heidkamp R, et al. Measuring the coverage of nutrition interventions along the continuum of care: time to act at scale, BMJ Global Health, 2019; 4 (Suppl 4): e001290. 
[19] Black RE, Allen LH, Bhutta ZA, et al. Maternal and child undernutrition: global and regional exposures and health consequences. The Lancet, 2008; 371 (9608): 243-260.

[20] Keenan JD, Gebresillasie S, Stoller NE, et al. Linear growth in preschool children treated with mass azithromycin distributions for trachoma: a cluster-randomized trial. PLOS ONE, 2019; neglected tropial diseases 13 (6): 1-12.

[21] Fenn B, Bulti AT, Nduna T, et al. An evaluation of an operations research project to reduce childhood stunting in a food-insecure area in Ethiopia. Public Health Nutrition, 2012; 15 (9): 1746-1754.

[22] Pridmore P and Hill RC. Addressing the underlying and basic causes of child undernutrition in developing countries: What works and why?'. Evaluation study, ministry of foreign affairs of Denmark, 2009; (2): 1-97.

[23] Moher D, Liberati A, Tetzlaff J, et al. Preferred Reporting Items for Systematic Reviews and Meta-Analyses: The PRISMA Statement. PLoS Medicine, 2009; 6 (7): e1000097.

[24] Evans N, Lasen M and Tsey K (2015) Effective Public Health Practice Project (EPHPP) Quality Assessment Tool for Quantitative Studies. 2015. Springer International Publishing: Springer Briefs in Public Health, 2015; 45-63. DOI 10.1007/978-3-319-17284-2

[25] Higgins, J. P. and Green, S. Cochrane Handbook for Systematic Reviews of Interventions, Cochrane Handbook for Systematic Reviews of Interventions: 2008. Cochrane Book Series. Edited by J. P. Higgins and S. Green. Chichester, UK: John Wiley \& Sons, Ltd. doi: 10.1002/9780470712184.

[26] EggerM, Smith GD, Schneider M, et al. Bias in meta-analysis detected by a simple, graphical test measures of funnel plot asymmetry. BMJ, 1997; 315 (7109): 629-34.

[27] Sachdev H, Gera $T$ and Nestel P. Effect of iron supplementation on physical growth in children: systematic review of randomised controlled trials. Public Health Nutrition, 2006; 9 (7): 904-920.

[28] Dossa R, Ategbo E, de Koning F, et al. Impact of iron supplementation and deworming on growth performance in preschool Beninese children. European Journal of Clinical Nutrition, 2001; 55 (4): 223-228.

[29] Pickering AJ, Djebbari H, Lopez C, et al. Effect of a community-led sanitation intervention on child diarrhoea and child growth in rural Mali: a cluster-randomised controlled trial. The Lancet Global Health, 2015; 3 (11): e701-e711.

[30] Hop LT and Berger J. Multiple micronutrient supplementation improves anemia, micronutrient nutrient status, and growth of Vietnamese infants: Double-blind, randomized, placebo-controlled trial. The Journal of Nutrition, 2005; 135 (3): 660S-665S.

[31] Alarcon K, Kolsteren PW, Prada AM, et al. Effects of separate delivery of zinc or zinc and vitamin A on hemoglobin response, growth, and diarrhea in young Peruvian children receiving iron therapy for anemia. Am J Clin Nutr, 2004; 80 (5): 1276-1282.

[32] Lopriore C, Guidoum Y, Briend A, et al. (2004) Spread fortified with vitamins and minerals induces catch-up growth and eradicates severe anemia in stunted refugee children aged, Am J Clin Nutr, 2004; 80: 973-981.

[33] Waswa LM, Jordan I, Herrmann J, et al. Community-based educational intervention improved the diversity of complementary diets in western Kenya: results from a randomized controlled trial. Public Health Nutrition, 2015; 18 (18): 3406-3419.

[34] Soliman AT, Al dabbagh MM, Habboub AH, et al. Linear growth in children with iron deficiency anemia before and after treatment. Journal of Tropical Pediatrics, 2009; 55 (5): 324-327.

[35] Perng W, Mora-Plazas M, Marin C, et al. Iron status and linear growth: a prospective study in school-Age children. European J Clin Nutr, 2013; 67 (6): 646-651.

[36] Ramakrishnan U, Nguyen P and Martorell R. Effects of micronutrients on growth of children under $5 \mathrm{y}$ of age: metaanalyses of single and multiple nutrient interventions. Am J Clin Nutr, 2009; 89: 191-203.

[37] MacDonald RS. The role of zinc in growth and cell proliferation, The Journal of Nutrition, 2000; 130 (5): 1500S$1508 \mathrm{~S}$.

[38] Vucic V, Berti C, Vollhardt C, et al. Effect of iron intervention on growth during gestation, infancy, childhood, and adolescence: a systematic review with meta-analysis. Nutrition Reviews, 2013; 71 (6): 386-401.

[39] Pasricha SR, Hayes E, Kalumba K, et al. Effect of daily iron supplementation on health in children aged 4-23 months: A systematic review and meta-analysis of randomised controlled trials. The Lancet Global Health, 2013; 1 (2): e77-e86.

[40] Smuts CM, Dhansay MA, Faber M, et al. Efficacy of multiple micronutrient supplementation for improving anemia, micronutrient status, and growth in South African infants. The Journal of Nutrition, 2005; 135 (3): 653S-659S.

[41] Dewey KG, Domello $\mathrm{M}$, Cohen RJ, et al. Iron supplementation affects growth and morbidity of breast-fed infants: results of a randomized trial in Sweden and Honduras. J. Nutr, 2002; 132: 3249-3255.

[42] Untoro J, Karyadi E, Wibowo L, et al. Multiple micronutrient supplements improve micronutrient status and anemia but not growth and morbidity of Indonesian infants: a randomized, double-blind, placebo-controlled trial. J. Nutr, 2005; 135: 639-645.

[43] Majumdar I, Paul P, Talib VH, et al. The effect of iron therapy on the growth of iron-replete and iron-deplete children. Journal of Tropical Pediatrics, 2003; 49 (2): 84-88.

[44] Thompson J, Biggs BA and Pasricha SR. Effects of daily iron supplementation in 2 to 5 -year-old children: systematic review and meta-analysis. Pediatrics, 2013; 131 (4): 739-753.

[45] Brown KH. Zinc and child growth: Commentary. International Journal of Epidemiology, 2003; 32 (6): 1103-1104.

[46] Osredkar, J. Copper and zinc, biological role and significance of copper/zinc imbalance. Journal of Clinical Toxicology, 2011; s3 (01): 1-18.

[47] Imdad A and Bhutta ZA. Effect of preventive zinc supplementation on linear growth in children under 5 years of age in developing countries. BMC Public Health, 2011; 11 (3): 377

[48] Das JK, Kumar R, Salam RA and Bhutta ZA. Systematic review of zinc fortification trials. Annals of Nutrition and Metabolism, 2013; 62 (s1): 44-56. 
[49] Adu-Afarwuah S, Lartey A, Brown KH, et al. Randomized comparison of 3 types of micronutrient supplements for home fortification of complementary foods in Ghana: effects on growth and motor development. Am J Clin Nutr, 2007; 86 (2): 412-420.

[50] Masuda K and Chitundu M. Multiple micronutrient supplementation using spirulinaplatensis and infant growth, morbidity, and motor development: Evidence from a randomized trial in Zambia. PLOS ONE, 2019; 14 (2): e0211693.

[51] Oelofse A, Van Raaij JMA, Benade AJS, et al. The effect of a micronutrient-fortified complementary food on micronutrient status, growth and development of 6- to 12-month-old disadvantaged urban South African infants. International Journal of Food Sciences and Nutrition, 2003; 54 (5): 399407.

[52] Shafique S, Sellen DW, Lou W, et al. Mineral- and vitaminenhanced micronutrient powder reduces stunting in full-term low-birth-weight infants receiving nutrition, health, and hygiene education : a cluster-randomized trial. Am J Clin Nutr, 2016; 103, p. 1357-69.

[53] Onyango AW, Borghi E, de Onis M, et al. Complementary feeding and attained linear growth among 6-23-month-old children. Public Health Nutrition, 2014; 17 (9): 1975-1983.

[54] Dangour AD, Watson L, Cumming O, et al. Interventions to improve water quality and supply, sanitation and hygiene practices, and their effects on the nutritional status of children. 2013, Cochrane Database of Systematic Reviews, (8). doi: 10.1002/14651858.CD009382.pub2.

[55] Rah JH, Cronin AA, Badgaiyan B, et al. Household sanitation and personal hygiene practices are associated with child stunting in rural India: a cross-sectional analysis of surveys. BMJ Open, 2015; 5 (2): 1-10.

[56] Ngure FM, Reid BM, Humphrey JH, et al. Water, sanitation, and hygiene (WASH), environmental enteropathy, nutrition, and early child development: Making the links. Annals of the New York Academy of Sciences, 2014; 1308 (1): 118-128. 